\title{
Elementary Echographic Estimation of Cardiac Output Independent of the Symmetry and Kinetic State of the Left Ventricle in Mitral Stenosis
}

\author{
Potential Application to the Determination of \\ the Circulation of Pregnancy
}

W.S. Seitz, M.D., M.S. Spiel, M.D., and K. Furukawa, M.D.*

\section{SUMmary}

An orifice equation is demonstrated which is independent of the symmetry and kinetic state of the left ventricle. The expression allows calculation of the cardiac output in conditions of mitral stenosis when the mitral valve area is known. This equation is $\dot{Q}=(1 / 21) \mathrm{R} \mathrm{A} \mathrm{T}^{2}$, where $\dot{\mathrm{Q}}=$ cardiac output in $\mathrm{ml} / \mathrm{min}, \mathrm{R}=$ heart rate, $\mathrm{A}=$ mitral valve area and $\mathrm{T}=$ diastolic filling period in sec/ min. Ten patients whose gynecologic or obstetric exam suggested a diagnosis of mitral stenosis were evaluated by conventional cardiac catheterization and M-mode echocardiography. Cardiac output computed using the new equation and the Fick principle corresponded at $\mathrm{r}=0.95, \mathrm{SE}=340 \mathrm{ml}, \mathrm{N}=10$. These results suggest that cardiac output may be conveniently estimated for serial studies by the readily measurable echographic variables of heart rate and diastolic filling period once the mitral valve area has been measured by conventional catheterization or bi-dimensional echographic methods.

\section{Additional Indexing Words:}

Cardiac output Echographic orifice formula Mitral stenosis

LEART disease during pregnancy is not a rare disorder, presenting in approximately $1 / 2$ to $2 \%$ of all cases. $^{1 !}$ Of this group, about 90 $95 \%$ of the heart disease is of rheumatic origin, predominantly mitral valve stenosis. ${ }^{21}$

As a result of the burden which pregnancy imposes on the heart vis-a-vis increased blood volume, cardiac output and heart rate, mitral stenosis can be

\footnotetext{
From the Division of Maternal and Fetal Medicine, Department of Obstetrics and Gynecology, University of Southern California Medical Center, Los Angeles, California, U.S.A. and the Second Department of Medicine, ${ }^{*}$ Kyoto Prefectural University of Medicine, Kyoto, Japan.

Address for reprint: W.S. Seitz, M.D., Gardiovascular Research Institute, University of California, School of Medicine, San Francisco, CA 94143, U.S.A.

Received for publication March 17, 1983.
} 
a particularly serious complicating condition during this period. Modest exercise can lead to tachycardia, a resulting decrease in diastolic filling period per diastolic minute and hence to an increased mitral valve gradient and capillary wedge pressure culminating in pulmonary edema with dyspnea. In patients whose pregnancy is complicated by mitral stenosis, serial evaluation of cardiac function is highly recommended to reduce both maternal and infant mortality. ${ }^{3}$ ) The traditional quantitive methods for assessing cardiac output are highly invasive in character and are thus inappropriate for sequential evaluations of this nature.

It would be desirable to be able to follow the cardiac output of such patients in a convenient, noninvasive manner. Current bi-dimensional echographic methods allow the approximate quantification of stroke volume and hence cardiac output at a clinically useful level. ${ }^{4,5)}$ Furthermore, Kronik ${ }^{(1)}$ et al have recently demonstrated that M-mode echocardiography can be expected to provide clinically useful estimates of stroke volume correlating with Fick principle measurements at levels of $r=0.84$ in the absence of wall motion irregularities. However, for sequential measurements in the same patient, variations in the angulation of the M-mode beam during the measurement sequence can lead to geometrical errors in cardiac volume.") Thus, an echographic method for the determination of cardiac output independent of the symmetry and dimensions of the left ventricle could provide a means for realizing serial measurements of this value.

A recent investigation ${ }^{8}$ ) has demonstrated an hydraulic orifice equation capable of determining mitral valve area in conditions of stenosis through variables measured solely by $\mathrm{M}$-mode or bi-dimensional echocardiography. This investigation suggested that an orifice equation having the same range of validity as the Gorlin formula is expressed as:

$$
\mathrm{A}=21 \dot{\mathbf{Q}} /\left(\mathrm{R} \mathrm{T}^{2}\right)
$$

where $\mathrm{A}$ is the mitral orifice area in $\mathrm{cm}^{2}, \dot{Q}$ is the cardiac output in $\mathrm{ml} / \mathrm{min}$, $\mathrm{R}$ is the heart rate and $\mathrm{T}$ is the diastolic filling period in sec/min. Equation (1) has been shown to correlate with the Gorlin formula for a series of resting state catheterization studies at a level of $r=0.94, N=46 .{ }^{8}$ )

This result suggests that equation (1) can provide a noninvasive method for estimating valvular obstruction in cases of mitral stenosis through echocardiography, contingent primarily upon the accuracy of the stroke volume measurement. Since mitral valve area is relatively constant, in the absence of active disease processes, over periods of several months or years; once the valve area is estimated by equation (1) or determined by conventional cardiac catheterization, equation (1) may be rearranged to yield a formula for cardiac 
output in terms of the known valve area and easily measured variables as:

$$
\dot{Q}=(1 / 21) \mathrm{R} \mathrm{A} \mathrm{T}^{2}
$$

This suggests that equation (2) could provide a convenient noninvasive method of following the absolute value and changes in cardiac output from the variables $R$ and $T$ using simple echographic measurements.

It was the purpose of the present investigation to test this concept in a series of gynecologic patients suffering from mitral stenosis, whose cardiac output was determined at cardiac catheterization, by conventional echographic methods and through the application of equation (2).

\section{Methods ahd Patients}

As a test of this formula, equation (2) was used to compute the cardiac output for a series of 10 patients whose initial gynecologic investigation suggested a diagnosis of mitral stenosis of sufficient degree to merit a catheterization study. The mean age of this group was 40 years (range $=28$ to 52). They had an average weight of $46 \mathrm{Kg}$ (range $=44$ to 50 ) and a mean body surface area of $1.4 \mathrm{~m}^{2}$ (range $=1.3$ to 1.48 ). In addition to the catheterization study, each patient underwent a standard M-mode echographic investigation. The only screening criterion applied to these patients was that the lesion be predominantly stenotic rather than regurgitant or mixed stenosis and insufficiency so that a meaningful comparison with classical invasive methods would be possible.

Paper strip chart echocardiograms were recorded on an Aloka SSD-90 echograph using a $2.25 \mathrm{MHz}, 1 \mathrm{~cm}$ diameter transducer at a paper speed of $10 \mathrm{~cm} / \mathrm{sec}$. The patients were studied supine with the transducer in the third or fourth intercostal space at the left sternal border. The ultrasound beam was positioned so as to identify the tip of the mitral valve leaflets and the transducer directed laterally and inferiorly so as to record the echoes from the posterior ventricular wall and the interventricular septum. The diastolic filling period from the separation to the coaptation of the anterior and posterior mitral valve leaflets was measured from the mitral valve echograms. These measurements were averaged over at least five cardiac cycles. The cnd-systolic and end diastolic dimensions were measured and the stroke volume computed according to the cubic formula.

All the patients were studied with right and left heart catheterization and the mitral valve area was computed by the standard Gorlin formula. The mitral valve pressure gradient was determined from simultaneous planigraphic measurements of left ventricular pressure and pulmonary capillary 
wedge pressure. The time delay resulting from retrograde transmission of wedge pressure pulse was taken, following Yang et al, ${ }^{9)}$ as $0.05 \mathrm{sec}$. The cardiac output was measured by the standard Fick principle method.

As an initial test of equation (2), the mitral valve area as computed by the Gorlin formula was combined with heart rate and diastolic filling period measured at cardiac catheterization to compute the cardiac output.

Next, equation (1) was used to compute mitral valve area from the echographic data of stroke volume, heart rate and diastolic filling period. This valve area was then used in equation (2) to calculate cardiac output with heart rate and diastolic filling period obtained from the catheterization study.

Finally, mitral valve area derived from the Gorlin formula at catheterization was combined with the echographically measured values of heart frequency and diastolic filling period in equation (2) to compute cardiac output. This value was compared with the cardiac output calculated by the product of the echographically determined stroke volume and heart rate and the Fick principle value.

\section{Results}

The principal finding of this study is the demonstration of a hydraulic orifice equation capable of enabling calculation of the cardiac output in patients with mitral stenosis using readily measurable echographic variables when the mitral valve area is known.

Evidence indicating the predictive capacity of this formula is presented in the form of 3 tables. Table I compares the results of cardiac output computed by equation (2) with cardiac output determined by the Fick principle method using catheterization data. This relationship is characterized by a correlation coefficient and standard error of $r=0.95$ and $S E=340 \mathrm{ml}$ / min, respectively.

Table II compares the results of a cardiac output calculation in which the mitral valve area was computed from noninvasive echographic data using equation ( 1 ) and then cardiac output calculated by applying equation (2) using catheterization data of heart rate and diastolic filling period with cardiac output determined by the Fick method. This comparison reveals a correlation coefficient and standard error of $r=0.79$ and $\mathrm{SE}=630 \mathrm{ml} / \mathrm{min}$, respectively.

In Table III, equation (2) is used to compute cardiac output from echographically derived values of heart rate and diastolic filling period and mitral valve area computed from the Gorlin formula. These values are compared with cardiac output as found by the product of the echographically derived 
Table I. Comparison of Cardiac Output Computed by the Fick Method and Equation (2) from Catheterization Data in Patients with Mitral Stenosis

\begin{tabular}{c|l|l|l|l|l|l}
\hline Case & RHY & $\mathrm{R}_{\mathrm{C}}$ & $\mathrm{A}_{\mathrm{G}}$ & $\mathrm{T}_{\mathrm{C}}$ & $\dot{\mathrm{Q}}_{\mathrm{F}}$ & $\dot{\mathrm{Q}}_{N}$ \\
\hline 1 & $\mathrm{SR}$ & 77 & 1.55 & 33.1 & 5.50 & 6.20 \\
2 & $\mathrm{AF}$ & 55 & 1.55 & 37.7 & 5.50 & 5.80 \\
3 & $\mathrm{SR}$ & 60 & 1.40 & 33.2 & 4.40 & 4.45 \\
4 & $\mathrm{SR}$ & 79 & 1.30 & 31.7 & 4.65 & 4.90 \\
5 & $\mathrm{SR}$ & 70 & 1.20 & 34.6 & 4.75 & 4.80 \\
6 & $\mathrm{SR}$ & 58 & 1.10 & 36.6 & 4.50 & 4.50 \\
7 & $\mathrm{SR}$ & 58 & 0.95 & 38.8 & 4.40 & 3.95 \\
8 & $\mathrm{AF}$ & 74 & 0.85 & 36.3 & 4.25 & 3.95 \\
9 & $\mathrm{SR}$ & 77 & 0.70 & 36.2 & 2.90 & 3.35 \\
10 & $\mathrm{SR}$ & 73 & 0.45 & 39.9 & 2.75 & 2.50 \\
\hline Mean & & & & & 4.36 & 4.44 \\
SE & & & & & & \\
r & & & & & & 0.34 \\
\end{tabular}

$\mathrm{R}_{\mathrm{C}}=$ heart rate at catheterization study; $A_{G}=$ mitral valve area from the Gorlin formula in $\mathrm{cm}^{2}$; $\mathrm{T}_{\mathrm{C}}=$ diastolic filling period in $\mathrm{sec} / \mathrm{min}$ at catheterization $; \dot{\mathrm{Q}}_{\mathrm{F}}=$ cardiac output from the Fick principle; $\dot{\mathrm{Q}}_{\mathrm{N}}=$ cardiac output from equation (2); $\mathrm{SE}=$ standard error of $\dot{\mathrm{Q}}_{\mathrm{N}}$ relative to $\dot{\mathrm{Q}}_{\mathrm{F}} ; \mathrm{r}=$ correlation coefficient; $\mathrm{RHY}=$ rhythm; $\mathrm{SR}=$ sinus rhythm; $\mathrm{AF}=$ atrial fibrillation.

Table II. Comparison of Cardiac Output Computed from the Fick Principle at Catheterization with Cardiac Output Determined from Equation (2) Using the Valve Area Found Noninvasively by M-mode Echo and Values of $T$ and $\mathrm{R}$ from the Catheterization Study

\begin{tabular}{|c|c|c|c|c|c|c|}
\hline Case & RHY & $\mathbf{R}_{\mathrm{C}}$ & $A_{E}$ & $\mathrm{~T}_{\mathrm{C}}$ & $\dot{\mathbf{Q}}_{\mathrm{F}}$ & $\dot{\mathrm{Q}}_{\mathrm{N}}$ \\
\hline 1 & $\mathrm{SR}$ & 77 & 1.30 & 33.1 & 5.50 & 5.20 \\
\hline 2 & $\mathrm{AF}$ & 55 & 1.40 & 37.2 & 5.50 & 5.10 \\
\hline 3 & SR & 60 & 1.20 & 33.1 & 4.40 & 3.80 \\
\hline 4 & SR & 79 & 1.45 & 30.9 & 4.65 & 5.50 \\
\hline 5 & SR & 70 & 1.00 & 35.3 & 4.75 & 4.00 \\
\hline 6 & SR & 58 & 0.85 & 36.1 & 4.50 & 3.15 \\
\hline 7 & SR & 58 & 1.05 & 35.8 & 4.40 & 4.35 \\
\hline 8 & $\mathrm{AF}$ & 74 & 0.95 & 33.3 & 4.25 & 4.40 \\
\hline 9 & SR & 77 & 0.70 & 34.2 & 2.90 & 3.35 \\
\hline 10 & SR & 73 & 0.50 & 35.3 & 2.75 & 2.50 \\
\hline Mean & & & & & 4.36 & 4.14 \\
\hline $\mathrm{SE}$ & & & & & & 0.63 \\
\hline$r$ & & & & & & 0.78 \\
\hline
\end{tabular}

$A_{E}=$ mitral valve area from equation (1) using echographic data. Remaining symbols as in Table I. 
Table III. Comparison of Cardiac Output Computed Echographically by the Product of Stroke Volume and Heart Rate with the Results of Equation (2) Using Area Computed from the Gorlin Formula with Data Derived from Catheterization

\begin{tabular}{c|c|c|c|c|c|c|c|c}
\hline Case & $\mathrm{RHY}$ & $\mathrm{V}_{\mathrm{E}}$ & $\mathrm{R}_{\mathrm{E}}$ & $\mathrm{A}_{\mathrm{G}}$ & $\mathrm{T}_{\mathrm{E}}$ & $\dot{\mathrm{Q}}_{\mathrm{N}}$ & $\dot{\mathrm{Q}}_{\mathrm{E}}$ & $\dot{\mathrm{Q}}_{\mathrm{F}}$ \\
\hline 1 & $\mathrm{AF}$ & 67.7 & 65 & 1.55 & 33.1 & 5.25 & 4.40 & 5.50 \\
2 & $\mathrm{AF}$ & 91.3 & 53 & 1.55 & 37.2 & 5.40 & 4.85 & 5.50 \\
3 & $\mathrm{NS}$ & 61.3 & 82 & 1.40 & 33.1 & 6.00 & 5.15 & 3.80 \\
4 & $\mathrm{NS}$ & 66.5 & 88 & 1.30 & 30.9 & 5.20 & 5.85 & 5.50 \\
5 & $\mathrm{NS}$ & 60.7 & 70 & 1.20 & 35.3 & 5.00 & 4.25 & 4.00 \\
6 & $\mathrm{NS}$ & 52.3 & 63 & 1.10 & 36.1 & 4.30 & 3.30 & 3.15 \\
7 & $\mathrm{NS}$ & 65.0 & 64 & 0.95 & 35.8 & 3.70 & 4.15 & 4.35 \\
8 & $\mathrm{AF}$ & 49.3 & 68 & 0.85 & 33.3 & 3.05 & 3.35 & 4.40 \\
9 & $\mathrm{NS}$ & 39.7 & 69 & 0.70 & 34.2 & 2.70 & 2.75 & 3.35 \\
10 & $\mathrm{NS}$ & 31.7 & 87 & 0.45 & 35.3 & 2.30 & 2.75 & 2.50 \\
\hline Mean & & & & & & 4.30 & 4.10 & 4.35 \\
$\mathrm{SE}$ & & & & & & & 0.65 & 0.79 \\
$\mathrm{r}$ & & & & & & & 0.86 & 0.69
\end{tabular}

$V_{E}=$ stroke volume computed by the cubic formula; $R_{E}=$ heart rate from echographic study; $A_{G}$ $=$ mitral valve area from the Gorlin formula; $T_{E}=$ diastolic filling period in sec/min from the echographic study; $\dot{Q}_{\mathrm{E}}=$ cardiac output from the product of stroke volume with heart rate; $\dot{\mathrm{Q}}_{\mathrm{N}}=$ cardiac output from equation $(2) ; \mathrm{RHY}=$ rhythm; NS=sinus rhythm ; $\mathrm{AF}=$ atrial fibrillation ; $\mathbf{Q}_{\mathrm{F}}$

= cardiac output from Fick principle. Remaining symbols as in Table I.

stroke volume and heart rate. These data correlate at a level given by $\mathrm{r}=0.86$ and $\mathrm{SE}=650 \mathrm{ml} / \mathrm{min}$. Additionally, we compared the cardiac output derived at catheterization with the echographically determined value via equation (2). This relationship is characterized by a correlation coefficient of $\mathrm{r}=0.69$ and a standard error of $\mathrm{SE}=790 \mathrm{ml} / \mathrm{min}$.

\section{Discussion}

The experiments summarized in Tables I to III assume, in concordance with the theory of Gorlin and Gorlin, ${ }^{11)}$ that the mitral valve area is a constant in a given patient in the absence of an acute rheumatic process. It is thus assumed that when the mitral valve area is determined by the Gorlin formula at catheterization, this value remains invariant over a wide range of hemodynamic states for many cardiac cycles. Under this assumption, repeated hemodynamic measurements via the Gorlin formula or another valid technique will yield approximately the same values within normal limits of measuremental error. It is thus possible to test an independent orifice formula using this calculated parameter as an intermediate value in an independent formula requiring area and other hemodynamic variables determined at any 
convenient catheterization study, including the study in which valve area is first measured.

The results expressed in Table I are consistent with the degree of correlation achieved by equation (1) in comparison with the Gorlin formula for a series of 46 cases studied previously. ${ }^{8}$. The current results suggest that when the mitral valve area is known, the cardiac output may be estimated through application of equation (2).

Previous studies have indicated that in the absence of significant asymmetric ventricular contraction, ventricular akinesis, hypokinesis or other wall motion irregularities, M-mode echography can be expected to yield stroke volume correlations with classical invasive methods in the range of $r=0.84$ to $\mathrm{r}=0.95 .{ }^{6), 10)}$

The application of equation (2) is independent of the state of symmetry of the left ventricle or wall motion irregularities once mitral valve area has been independently determined. Cardiac output may then be calculated by using the readily measurable variables of heart rate and diastolic filling period. Equation (2) could be expected to apply in mitral stenosis whenever the mitral valve leaflet motion can be recorded with $\mathrm{M}$-mode echocardiography, including using the currently available portable devices.

Application of this principle could have an impact in patient management in that a simplified method of following cardiac output in conditions of stenosis could lead to a more rational basis for the administration of therapeutic measures or the proscription of activity. Furthermore, once cardiac output is known, the pulmonary capillary wedge pressure may be estimated by solving the Gorlin equation for this quantity according to the original method of Gorlin and Gorlin. ${ }^{11)}$

The results of Table II suggest that when equation (1) is used to compute mitral valve area from M-mode echocardiographic measurements, cardiac output data computed at another time through knowledge of both diastolic filling period and heart rate correlate at a level which reflects the uncertainties in an M-mode echographic estimation of stroke volume and hence valve area via equation (1). Despite the fact that the correlation with classical invasive techniques is modest when valve area is determined by M-mode echography, it should be noted that for the purpose of following the cardiac output in an individual patient, changes in the cardiac output are not affected by an error in the initial stroke volume determination, but are, rather, affected only by errors in the measurement of diastolic filling period and heart rate. Each of these variables may be measured to a high degree of accuracy by $\mathrm{M}$ mode echocardiography. Thus, errors in the determination of changes in cardiac output are proportional only to these errors and not to an error in the 
estimate of stroke volume.

These considerations are further exemplified by the results shown in Table III. These data suggest that the discrepancy in correlation between the two methods of computation of cardiac output may be a result of errors in the measurement of stroke volume. Here, cardiac output computed by the expression $\dot{Q}=(1 / 21) R \mathrm{~A} \mathrm{~T}^{2}$ shows a higher degree of correlation than the results of the invasive study in which the area used in equation (2) derives from an M-mode estimate of valve area via equation (1).

Comparison of the correlation coefficients and standard errors of the data in the 3 tables suggests that cardiac output can be followed echographically when the valve area is known through application of equation (2). Furthermore, the current evidence indicates that the level of accuracy achieved by the application of equation (2) may be greater than that achieved by computation using the product of stroke volume and heart rate in patients with mitral stenosis.

In the present study we attempted to minimize the interval separating the catheterization and echographic studies in a manner consistent with the patient's comfort and the schedule of the investigators. In no case was this interval greater than 2 weeks. However, due to short term variations in minute volume, the comparison between the cardiac output determined by the Fick principle and the echographically derived value of this parameter could not be expected to show a significant correlation. The computed value of $r=0.69$ demonstrates this concept.

The strong dependence of equation (2) on heart rate and diastolic filling period suggests that in arrhythmic states, averaging of many cardiac cycles and diastolic flow intervals would be desirable. In conditions of regurgitation, equation (2) cannot be expected to yield the forward cardiac output, but rather, the total forward and reverse volume flow.

In conclusion, the orifice equation:

$$
\dot{\mathrm{Q}}=(1 / 21) \mathrm{R} \text { A T } \mathrm{T}^{2}
$$

where $\dot{Q}$ is the cardiac output in $\mathrm{ml} / \mathrm{min}, \mathrm{R}$ is the heart rate, $\mathrm{A}$ is the mitral valve area and $T$ is the diastolic filling period in sec/min is capable of estimating cardiac output in conditions of mitral stenosis when the mitral valve area is known. Further, these results suggest that equation (2) may offer the potential to estimate serial changes in the circulation during pregnancy once the mitral valve area has been estimated via standard bi-dimensional echography through parameters measurable via elementary M-mode echographic techniques. 


\section{REFERENCES}

1. Benson RC: Gurrent Obstetric Gynecologic Diagnosis and Treatment, Lange Medical Publications, Los Altos, p 725-26, 1976

2. Sokolow M, McIlroy MB: Clinical Cardiology, Lange Medical Publications, Los Altos, p 610-17, 1977

3. Burch GE: Heart disease and pregnancy. Am Heart J 93: 104, 1977

4. Schiller NB, Acquatella $H$, Ports TA et al: Left ventricular volume from paired biplane twodimensional echocardiography. Circulation 60: 547, 1979

5. Silverman NH, Ports TA, Snider AR, Schiller NB, Carlson E, Heilborn DC: Determination of left ventricular volume in children. Echocardiographic and angiographic comparisons. Circulation 62: 548, 1980

6. Kronik G, Slany J, Mösslacher H: Comparative value of eight M-mode echocardiographic formulas for determining left ventricular stroke volume. Circulation 60: 1308, 1979

7. Wong M, Shah PM, Taylor RD: Reproducibility of left ventricular internal dimensions with M-mode echocardiography. Effects of heart size, body position and transducer angulation. Am J Cardiol 47: 1067, 1981

8. Seitz WS, Operschall EJ: Noninvasive determination of the mitral valve area in stenosis: a computational model and correlation with autopsy and open heart measurements. Cardiovase Res 14: 223, 1980

9. Yang SS, Bentivoglio LG, Maranhad V, Goldberg H: Basic measurements and calculations. in From Cardiac Catheterization Data to Hemodynamic Parameters, 2nd Ed, FA Davis, Philadelphia, p 42-45, 1978

10. Popp RL, Harrison DC: Ultrasonic cardiac echography for determining stroke volume and valvular regurgitation. Circulation 41: 493, 1970

11. Gorlin R, Gorlin SG: Hydraulic formula for calculation of the area of the stenotic mitral valve, other cardiac valves, and central circulatory shunts. Am Heart J 41: 1, 1951 